\title{
Possible roles of CD73 (ecto-5'-nucleotidase) and clinical significance in metastasis human cancer
}

\author{
Marco Antonio Lacerda-Abreu ${ }^{1,2 *}$ and José Roberto Meyer-Fernandes ${ }^{1,2 *}$ \\ ${ }^{1}$ Institute of Medical Biochemistry Leopoldo de Meis; Federal University of Rio de Janeiro, RJ, Brazil \\ ${ }^{2}$ National Institute of Science and Technology in Structural Biology and Bioimaging, Rio de Janeiro, RJ, Brazil
}

\begin{abstract}
CD73, also designated ecto-5'-nucleotidase is one kind of ecto-nucleotidase that plays a critical role in tumor development. CD73 overexpression is well established in the literature for various cancers. However, increasing studies have shown a strong relationship between CD73 for metastatic processes. In this review, we summarize major roles of CD73 expression for the development of metastases in different tumor tissues and possible therapeutic target for metastasis. In addition, we list possible CD73 functions, either by adenosine generation as adenosine receptor agonist (metastatic signaling modulator) or inorganic phosphate (Pi) generation as a substrate for the high bioenergetic metastatic processes demand.
\end{abstract}

\section{Introduction}

Metastasis is a complex process and a major contributor of death in cancer patients [1]. The metastatic process is thought to consist of a number of distinct steps: 1) Invasion requires neoplastic epithelial cells to lose cell-cell adhesion and to gain motility for migration and cell adhesion to matrix extracellular (MEC), this represents a shift toward the mesenchymal state for epithelial to mesenchymal transition (EMT), which enables them to invade the adjacent tissue [2]. 2) On intravasation, tumor cells penetrate through the blood endothelium or lymphatic vessels to enter the systemic circulation and must be able to survive this stressful environment. 3) Survivors cell extravasate through the capillary endothelium at distal sites. 4) In the new host environment, an even smaller subset of such metastasizing cells succeeds in proliferating from minimum growths into malignant secondary tumors [3].

The mitogen-activated protein kinase (MAPK) are the most common signaling cascade for metastatic progression [1]. This cascade starts with the binding of an extracellular mitogenic ligand to the membrane receptor (ie EGFR, PDGFR) leading to activation of Ras (a GTPase) which activates series of MAPKs via MAP3K, MAP2K, MAPK and finally activation of metastatic transcription factors [1].

In solid tumors, ATP is released into the extracellular space through the channels and panexin connections, a higher concentration than in healthy tissues, due to cell death in the tumor nucleus, metabolic or hypoxic stress [4]. Ecto-nucleotidases are responsible for ATP hydrolysis generating ADP, AMP, adenosine and $\mathrm{Pi}$, for three main classes of ectonucleotidases: 1) Ecto-nucleoside triphosphate diphosphohydrolase (CD39, E-NTPDase) generating ADP and AMP from ATP hydrolysis; 2) Ecto-nucleotide pyrophosphatase/phosphodiesterases, hydrolysis of ATP to AMP; 3) Ecto-5'-nucleotidase, generating adenosine and Pi from 5'AMP hydrolysis [5-6]. Regarding Ecto-5'-nucleotidase, overexpression is well established in the literature for various cancers [6].

In this review, we shown the main works on CD73 expression in the development of metastasis in different tumor tissues. In addition, we seek to understand mechanically how the CD73 enzyme regulates metastatic processes.

\section{CD73 in several metastatic cancers}

There is a consensus on high CD73 expression in different cancer tissues [6]. In recent years, CD73 expression has been associated with metastatic processes. In non-small cell lung cancer (NSCLC), silenced CD73 cell lines A549 and H226 showed significant inhibition of cell migration and invasion [7]. In melanoma cell lines (A375 cells), an enzymatic and non-enzymatic function of ecto-5'-nucleotidase (CD73) in migration and invasion has been demonstrated [8]. Regardless of its enzymatic role, it is known that CD73 can mediate cell-cell adhesion by being a co-receptor in $\mathrm{T}$ cell activation or regulating cell interaction with extracellular matrix (ECM) components and migration therein [8-9].

Immunohistochemical analysis from melanoma patients samples, showed elevated CD73 expression in metastatic patients. In addition, was demonstrated an association of decreased survival with CD73 expression, whereas CD73 in tumor infiltrating mononuclear cells was significantly associated with survival improvement [10].

A study using human cervical cancer cell lines (HeLa and SiHa) overexpressing CD73 showed a 50\% increase in cell migration.In the same study, ACPP $(50 \mu \mathrm{M})$, a specific inhibitor of CD73 enzyme activity has no effect on cell migration, suggesting a regulation independent of enzymatic activity. On the other hand, high concentration of adenosine $(100 \mu \mathrm{M}-1 \mathrm{mM})$ showed a decrease cell migration, suggesting a distinct

*Correspondence to: Marco Antonio Lacerda-Abreu and José Roberto MeyerFernandes, Instituto de Bioquímica Médica, Universidade Federal do Rio de Janeiro, CCS, Cidade Universitária, Rio de Janeiro, RJ 21941-590, Brazil, Tel: +55-21-3938-6781; Fax: +55-21-2270-8647; E-mail: meyer@bioqmed.ufrj.br

Key words: ecto-5'-nucleotidase, CD73, metastasis, adenosine-receptor, $\mathrm{Pi}$ transporters

Received: January 23, 2020; Accepted: February 13, 2020; Published: February 17,2020 
mechanism for adenosine receptors [11]. This latter observation was previously confirmed in PC-3 cells (human prostate carcinoma) and MDA-MB-231 cells (human breast cancer), a significant reduction in cell invasion with 1-50 $\mu \mathrm{M}$ adenosine [12].

Regarding breast cancer, a distinct group using T47-D cells, observed a stimulation of cell invasion, migration and adhesion (slightly more than twice) with adenosine $(100 \mu \mathrm{M})$ and an inhibition with ACPP $(12 \mu \mathrm{M})$ [13]. Suggesting that CD73 may facilitate the metastatic process of human breast cancer cells through its enzyme activity of generating adenosine [13], as discussed later.

Hepatocellular carcinoma cell lines (HCCLM3 and SMMC7721) with CD73 silenced showed an inhibition of cell invasion and migration. In those same cells, by deleting the gene in xenograft model groups, the incidence of intrahepatic or lung metastasis was lower compared to the control and the opposite was observed when overexpressing the CD73 gene [14]. Moreover, CD73, E-Cadherin, and N-Cadherin expressions in hepatocellular carcinoma samples showed that in tissues with high CD73 expression levels have mesenchymal phenotype and samples with low level CD73 expression has phenotype more epithelial associating the CD73 expression with epithelial-mesenchymal transition [14].

\section{CD73 acting as possible metastatic clinical targets}

Some studies have shown different ways to impair CD73 function associated with damage to metastatic processes. The applicability of anti-CD73 monoclonal antibody (mAb) to breast cancer therapy in mouse models has been demonstrated [15]. The study inoculated mouse metastatic cell line (4T1.2) into BALB/C mice. In mice previously treated with anti-CD73 showed a significant reduction in lung metastasis compared to mice treated with the vehicle [15]. In other experiments of a distinct group, indicated that anti-CD73 could inhibit cell migration and invasion in human triple-negative breast cancer (MDA-MB-231 and MDA-MB-468 cells) and mouse 4T1 cell lines. In vivo analysis, anti-CD73 mAb could significantly inhibit lung metastasis of $4 \mathrm{~T} 1$ cells in a mouse xenograft model [16]. This result set suggests the possible applicability of anti-CD73 for metastasis treatment, however, more studies need to be done regarding this applicability.

A new CD73 applicability has been suggested using tiamulin hydrogen fumarate (THF; $25 \mu \mathrm{g} / \mathrm{mL}$ ), a classic veterinary antibiotic showed a significant reduction in CD73 enzyme activity, whereas migration and invasion in MDA-MB-231 or T41 cells were reduced by THF. This trend was reversed by treatment with adenosine, suggested that THF inhibited cell migration and invasion by CD73 activity decreased [17].

One study using mouse models of breast cancer has demonstrated that CD73 overexpression in tumor cells conferred chemoresistance to doxorubicin, by suppressing adaptive antitumor immune responses via activation of A2A adenosine receptors [18]. Stagg and colleagues, shown that targeted therapy against CD73 can trigger adaptive antitumor immunity and inhibit metastasis of breast cancer [15]. In addition, Bowser, et al. demonstrated CD73 deficiency led to a loss of epithelial barrier function endometrial, and pharmacological CD73 inhibition increased in vitro migration and invasion of endometrial carcinoma cells [19]. These observations demonstrate the importance of caution in inhibiting CD73 and the possible impacts on non-tumor tissues in the human body.

\section{Adenosine release function for metastasis}

Ecto 5'-nucleotidase hydrolyzes AMP into adenosine, which is a major source of adenosine for adenosine (P1) receptors, whereas ATP is common substrate for $\mathrm{P} 2$ receptors. Together, acts a complex role in pathological and physiological functions of the body [20]. In this study, we will focus solely on adenosine receptor (P1) function as the main trigger for metastatic processes.

There are four subtypes of adenosine receptors (ARs), named $\mathrm{A} 1, \mathrm{~A} 2 \mathrm{~A}, \mathrm{~A} 2 \mathrm{~B}$ and $\mathrm{A} 3$, all of which are $\mathrm{G}$ protein-coupled receptors (GPCRs) [5]. Both A1 and A3 receptors decrease cAMP levels, whereas $\mathrm{A} 2 \mathrm{~A}$ and $\mathrm{A} 2 \mathrm{~B}$ increase cAMP contents. Adenosine receptors activate MAPK pathways and, in some cells, $\mathrm{A} 1, \mathrm{~A} 3$, and $\mathrm{A} 2 \mathrm{~B}$ receptors direct phosphatidylinositol 3-kinase (PI3K), overall acting on different pathways, mainly involved in metastatic processes [21]. For example, a group using a non-selective adenosine receptor antagonist (aminophylline) or a selective A2B receptor antagonist (ATL801) had a significant reduction by $85 \%$ breast cancer cells from mammary fat to lung [22]. In addition, Beavis, et al. shown that A2A receptor blockade potentially suppresses $\mathrm{CD}^{2} 3^{+}$tumor metastasis and Young, et al. demonstrated Co-inhibition of CD73 and $\mathrm{A}_{2 \mathrm{AR}}$ adenosine signaling improves anti-tumor immune responses [23-24].

\section{$P i$ release function for metastasis}

Ecto-5'-nucleotidases catalyses the hydrolysis of 5' carbon esterified phosphate from ribose or deoxyribose. High nucleotidase expression in tumor tissues has commonly been associated with extracellular adenosine release. Little is discussed about the contribution of $\mathrm{Pi}$ released from hydrolysis to these metastatic processes [6].

$\mathrm{Pi}$ is a fundamental component of phospholipids and the nucleotides that form DNA and RNA. Inorganic phosphate is associated with energy metabolism, either in the form of ATP or in its free form as a substrate for the intermediates of metabolic pathways [25].

It was observed that cell growth in a medium with a high $\mathrm{Pi}$ ( 3 or $5 \mathrm{mM} \mathrm{Pi})$ compared to normal $\mathrm{Pi}(1 \mathrm{mM} \mathrm{Pi})$ concentration displayed a greater migratory capacity, could be explained by expression of osteopontin regulated by Forkhead Box Protein C2 (FOXC2) [26].

$\mathrm{Pi}$ enters the cells via $\mathrm{Na} / \mathrm{Pi}$ cotransporters. These cotransporters constitute two large families of $\mathrm{Na}^{+}$Dependent inorganic phosphate transporters that have been characterized in mammals, namely, SLC20 and SLC34. Regarding the SLC34 family, consists of three members, namely, NaPi-IIa (SLC34A1), NaPi-IIb (SLC34A2), and NaPi-IIc (SLC34A3) [27]. Several studies suggest that NaPi-IIb (SLC34A2) is upregulated in tumor cells and thus have been considered to be important promoters of tumor progression [25]. Russo-Abrahão, et al. showed the Pi transport level higher in cells breast cancer cells with greater metastatic potential such as MDA-MB-231 compared to other breast cancer cells (MCF-7 and T47-D). In addition, inhibition of $\mathrm{Na}^{+}$ dependent Pi transport in those cells significant reduced tumor cell migration and adhesion [28]. Lacerda-Abreu, et al. 2019 demonstrated in MDA-MB-231 cells, a low affinity $\mathrm{H}^{+}$-dependent $\mathrm{Pi}$ transporter is related to cell migration and adhesion process, especially in high $\mathrm{Pi}$ concentrations. Also was shown that MDA-MB-231 cells tend to revert from mesenchymal to epithelial features when the $\mathrm{H}^{+}$-dependent $\mathrm{Pi}$ transport is inhibited [29].

\section{Conclusion}

The development of metastases represents the fortuitous survival and growth of very few neoplastic cells. In this review, we summarize 
the importance of CD73 for metastatic processes in different tumor tissues and possible use as a therapeutic target for metastasis. This would occur by mechanisms independent of enzymatic function, in which CD73 may perform a receptor-like function by controlling metastatic signaling. Regarding the enzymatic function of CD73 and its generated products (adenosine and $\mathrm{Pi}$ ), adenosine binds at $\mathrm{P} 1$ receptor, generally acts on the MAPK pathway, the main signaling pathway involved in signaling metastatic processes. Inorganic phosphate possibly acts as substrate for the $\mathrm{Na}^{+}$-dependent or $\mathrm{H}^{+}$-dependent $\mathrm{Pi}$ transporters, providing more Pi intracellular for metastatic bioenergetic demand.

\section{Acknowledgements and Funding}

This work was supported by research grants Brazilian National Council for Scientific and Technological Development (CNPq), The State of Rio de Janeiro Research Foundation (FAPERJ), and the Coordination for the Improvement of Higher Education Personnel (CAPES) supported this investigation.

\section{References}

1. Khan I, Steeg PS (2017) Metastasis suppressors: functional pathways. Laboratory Investigation 98: 198-210.

2. Yang J, Mani SA, Donaher JL, Ramaswamy S, Itzykson RA, et al. (2004) Twist, a master regulator of morphogenesis, plays an essential role in tumor metastasis. Cell 117: 927-939. [Crossref]

3. Fidler IJ (2003) The pathogenesis of cancer metastasis: the 'seed and soil' hypothesis revisited. Nat Rev Cancer 3: 453-458. [Crossref]

4. Allard B, Longhi MS, Robson SC, Stagg J, et al. (2017) The ectonucleotidases CD39 and CD73: Novel checkpoint inhibitor targets. Immunol Rev 276: 121-144. [Crossref]

5. Stagg J, Smyth MJ (2010) Extracellular adenosine triphosphate and adenosine in cancer. Oncogene 29: 5346-5358. [Crossref]

6. Lacerda-Abreu MA, Meyer-Fernandes JR (2019) Release of Inorganic Phosphate into the Tumor Environment: Possible Roles of Ecto-Nucleotidases and Ecto-Phosphatases. Nov Appro in Can Study 3: NACS.000568.

7. Zhu J, Zeng Y, Li W (2017) CD73/NT5E is a target of miR-30a-5p and plays an important role in the pathogenesis of non-small cell lung cancer. Mol Cancer 16: 34. [Crossref]

8. Sadej R, Skladanowski AC (2012) Dual, enzymatic and non-enzymatic, function of ecto-5'-nucleotidase (eN, CD73) in migration and invasion of A375 melanoma cells. Acta Biochim Pol 59: 647-652. [Crossref]

9. Airas L, Hellman J, Salmi M, Bono P, Puurunen T, et al. (1995) CD73 is involved in lymphocyte binding to the endothelium: characterization of lymphocyte-vascular adhesion protein 2 identifies it as CD73. J Exp Med 182: 1603-1608. [Crossref]

10. Airas L, Hellman J, Salmi M, Bono P, Puurunen T, et al. (1995) CD73 is involved in lymphocyte binding to the endothelium: characterization of lymphocyte-vascular adhesion protein 2 identifies it as CD73. J Exp Med 182: 1603-1608. [Crossref]

11. Monteiro I, Vigano S, Faouzi M, Treilleux I, Michielin O, et al. (2018) CD73 expression and clinical significance in human metastatic melanoma. Oncotarget 9: 26659-26669. [Crossref]

12. Gao ZW, Wang HP, Lin F, Wang X, Long M, et al. (2017) CD73 promotes proliferation and migration of human cervical cancer cells independent of its enzyme activity. $B M C$ Cancer 17: 135. [Crossref]
13. Virtanen SS, Kukkonen-Macchi A, Vainio M, Elima K, Härkönen PL, et al. (2014) Adenosine inhibits tumor cell invasion via receptor-independent mechanisms. Mol Cancer Res 12: 1863-1874. [Crossref]

14. Ma XL, Shen MN, Hu B, Wang BL, Yang WJ, et al. (2019) CD73 promotes hepatocellular carcinoma progression and metastasis via activating PI3K/AKT signaling by inducing Rap1-mediated membrane localization of $\mathrm{P}^{110 \mathrm{I}^{2}}$ and predicts poor prognosis. J Hematol Oncol 12: 37. [Crossref]

15. [Crossref] Stagg J1, Divisekera U, McLaughlin N, Sharkey J, Pommey S, et al. (2010) Anti-CD73 antibody therapy inhibits breast tumor growth and metastasis. Proc Natl Acad Sci U S A 107: 1547-1552.

16. [Crossref] Qiao Z1, Li X2, Kang N3, Yang Y4, Chen C5, et al. (2019) A Novel Specific Anti-CD73 Antibody Inhibits Triple-Negative Breast Cancer Cell Motility by Regulating Autophagy. Int J Mol Sci 20.

17. Yang X, Pei S, Wang H, Jin Y, Yu F (2017) Tiamulin inhibits breast cancer growth and pulmonary metastasis by decreasing the activity of CD73. BMC Cancer 17: 255. [Crossref]

18. Loi S, Pommey S, Haibe-Kains B, Beavis PA, Darcy PK, et al. (2013) CD73 promotes anthracycline resistance and poor prognosis in triple negative breast cancer. Proc Natl Acad Sci U S A 110: 11091-11096. [Crossref]

19. Bowser JL, Blackburn MR, Shipley GL, Molina JG, Dunner K Jr, et al. (2016) Loss of CD73-mediated actin polymerization promotes endometrial tumor progression. J Clin Invest 126: 220-238. [Crossref]

20. Iqbal J (2019) Ectonucleotidases: Potential Target in Drug Discovery and Development Mini Rev Med Chem 19: 866-869. [Crossref]

21. Arab S1,2, Hadjati J3 (2019) Adenosine Blockage in Tumor Microenvironment and Improvement of Cancer Immunotherapy. Immune Netw 19: e23. [Crossref]

22. Cekic C, Sag D, Li Y, Theodorescu D, Strieter RM, et al. (2012) Adenosine A2B receptor blockade slows growth of bladder and breast tumors. J Immunol 188: 198205. [Crossref]

23. Beavis PA, Divisekera U, Paget C, Chow MT, John LB, et al. (2013) Blockade of A2A receptors potently suppresses the metastasis of CD73+ tumors. Proc Natl Acad Sci U S A 110: 14711-14716. [Crossref]

24. Young A, Ngiow SF1, Barkauskas DS2, Sult E3, Hay C3, et al. (2016) Co-inhibition of CD73 and A2AR Adenosine Signaling Improves Anti-tumor Immune Responses. Cancer Cell 30: 391-403. [Crossref]

25. Lacerda-Abreu MA, Russo-AbrahÃ£o T, Monteiro R de Q, Rumjanek F D, MeyerFernandes JR (2018) Inorganic phosphate transporters in cancer: Functions, molecular mechanisms and possible clinical applications. Biochim Biophys Acta Rev Cancer 1870: 291-298.

26. Lin Y, McKinnon KE, Ha SW, Beck GR Jr (2015) Inorganic phosphate induces cancer cell mediated angiogenesis dependent on forkhead box protein $\mathrm{C} 2$ (FOXC2) regulated osteopontin expression. Mol Carcinog 54: 926-934. [Crossref]

27. Forster IC, Hernando N, Biber J, Murer H (2013) Phosphate transporters of the SLC20 and SLC34 families. Mol Aspects Med 34: 386-395. [Crossref]

28. Russo-Abrahão T, Lacerda-Abreu MA, Gomes T (2018) Characterization of inorganic phosphate transport in the triple-negative breast cancer cell line, MDA-MB-231. PLoS One 13: e191270. [Crossref]

29. Lacerda-Abreu MA, Russo-Abrahão T, Cosentino-Gomes D, Nascimento MTC Carvalho-Kelly LF, et al. (2019) H+-dependent inorganic phosphate transporter in breast cancer cells: Possible functions in the tumor microenvironment. Biochim Biophys Acta Mol Basis Dis 1865: 2180-2188. [Crossref]

Copyright: (C2020 Lacerda-Abreu MA. This is an open-access article distributed under the terms of the Creative Commons Attribution License, which permits unrestricted use, distribution, and reproduction in any medium, provided the original author and source are credited. 